\title{
Heterogeneous clusters as a model system for the study of ionization dynamics within tampered samples
}

\author{
B. Ziaja, ${ }^{1,2,{ }^{*}}$ H. N. Chapman,,${ }^{1,3}$ R. Santra, ${ }^{1,4}$ T. Laarmann, ${ }^{5}$ E. Weckert, ${ }^{5}$ C. Bostedt,${ }^{6}$ and T. Möller ${ }^{7}$ \\ ${ }^{1}$ Center for Free-Electron Laser Science, Deutsches Elektronen-Synchrotron, Notkestrasse 85, D-22607 Hamburg, Germany \\ ${ }^{2}$ Institute of Nuclear Physics, Polish Academy of Sciences, Radzikowskiego 152, PL-31-342 Kraków, Poland \\ ${ }^{3}$ Department of Physics, University of Hamburg, Luruper Chaussee 149, D-22761 Hamburg, Germany \\ ${ }^{4}$ Department of Physics, University of Hamburg, Jungiusstrasse 9, D-20355 Hamburg, Germany \\ ${ }^{5}$ Hamburger Synchrotronstrahlungslabor, Deutsches Elektronen-Synchrotron, Notkestrasse 85, D-22607 Hamburg, Germany \\ ${ }^{6}$ Linac Coherent Light Source, SLAC National Accelerator Laboratory, 2575 Sand Hill Road, Menlo Park, California 94025, USA \\ ${ }^{7}$ Institut für Optik und Atomare Physik, Technische Universität Berlin, D-10623 Berlin, Hardenbergstrasse 36, Germany
}

(Received 31 May 2011; published 29 September 2011)

\begin{abstract}
Tampering of a sample with a layer of another material is a promising technique to slow down the expansion dynamics within laser irradiated samples, with sound implications for single-particle diffraction imaging. Ideally, if an imaged object is covered by a layer of another material, during the irradiation this layer will be primarily ionized and will expand fast due to Coulomb repulsion, whereas the object located within the net neutral core will expand more slowly (hydrodynamically). We investigate the details of the electronic damage within the tampered samples during their irradiation with an intense extreme ultraviolet (EUV) pulse. We study heterogeneous clusters composed of noble gas atoms, Xe and Ar, for which chemical-bond effects can be neglected. Using a fully nonequilibrium kinetic equation code, we demonstrate the influence of cluster composition on ionization dynamics; in particular, on the electronic damage. The results are obtained for the wavelength of $32 \mathrm{~nm}$, which is available at the free-electron laser in Hamburg (FLASH) facility, but our conclusions can also have implications for samples with a more complex structure and irradiated at a much shorter wavelength.
\end{abstract}

DOI: 10.1103/PhysRevA.84.033201

PACS number(s): 36.40.-c, 41.60.Cr, 52.50.Jm, 52.30.-q

\section{INTRODUCTION}

Atomic clusters are physical objects of nanometer size, consisting of up to tens of thousands of closely packed atoms. Their physical properties put them on the border between solid state and gas phase. Clusters are excellent objects to test the dynamics of samples irradiated with shortwavelength radiation from free-electron lasers (FELs) [1-10] and from high-harmonic-generation sources [11]. By studying the dependence of the cluster response on the cluster size, the influence of the atomic and condensed-matter effects on the ionization dynamics can be explored. Cluster studies are important for planned experiments with FELs within solid state physics, material sciences, and for studies of the extreme states of matter [12-14]. In particular, estimates of the ionization, thermalization, and expansion timescales that can be obtained from cluster studies are needed to explore the limits for experiments on single-particle diffraction imaging [15-20].

Single-particle diffraction imaging proceeds through elastic scattering of x-ray photons from atoms and ions and recording the obtained diffraction image. An ultrashort pulse of intense $\mathrm{x}$-ray radiation can record an image of the particle before it is destroyed by radiation damage. However, the pulse parameters have to be adjusted to the specific radiation tolerance of the particle depending on its composition and size. If the pulse fluence is too high, the radiation damage progresses too fast. High charges are created within the sample. This lowers the imaging signal as highly stripped ions have lower scattering factors. If the sample is highly ionized, ions can even start

\footnotetext{
*ziaja@mail.desy.de
}

to move apart during the pulse due to strong repulsive forces between them. The ion displacement additionally disturbs the image. Also, elastic scattering from quasifree electrons within the sample contribute to the signal as a background, reducing contrast in the scattering pattern.

On the other hand, if the pulse fluence is too low, radiation damage will progress more slowly but the imaging signal recorded from elastic scattering will be too noisy for structural information to be extracted. Therefore, imaging requires a reliable characterization of the pulse fluence limits within which an image of good quality can be recorded.

For small samples the proper adjustment of the imaging pulse parameters may not be possible, as the radiation damage progresses fast, even at relatively low fluences. In this case, tampering of the sample with a layer of another material as proposed in [19] and explored in [14,21-23] is a promising technique to slow down the sample expansion. During the irradiation of the sample, a layer structure consisting of a core of zero net charge and a positively charged outer shell is formed $[17,24,25]$. The outer shell (consisting of unscreened ions) expands fast, due to Coulomb repulsion among the ions. Within the core, the ion charges are screened by quasifree electrons, and the core expands slowly (hydrodynamically). Therefore, if during imaging an imaged object were covered by a layer of another material, this layer would be primarily ionized and expand fast, whereas the object located within the net neutral core would expand much more slowly.

Originally, the tampering technique aimed at slowing down the expansion of imaged samples. However, impact ionization by electrons from the tamper (electronic damage) leads to the production of high charges within the core. These charges are screened by quasifree electrons confined within the core but 

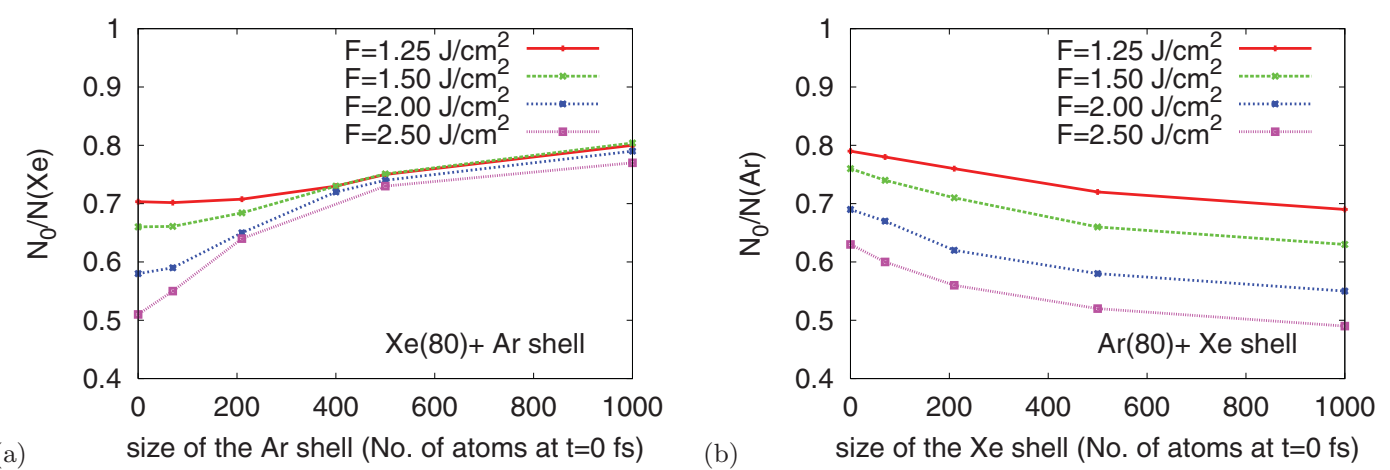

FIG. 1. (Color online) Content of neutral atoms within the Xe/Ar cores as a function of the thickness of the tampering layer for (a) $\mathrm{Xe}_{80}$ covered with an Ar layer, and (b) $\mathrm{Ar}_{80}$ covered with an Xe layer. The pulses were temporally rectangular, with a fluence of $F=1.25$ to $2.5 \mathrm{~J} / \mathrm{cm}^{2}$. The results were obtained with Boltzmann solver simulations.

their contribution to the elastic scattering signal is much lower than that obtained from the scattering from neutral atoms. Here we investigate the details of the electronic damage within the tampered samples (heterogeneous clusters) during their interaction with the FEL pulse by estimating the content of neutral atoms in the core. This content is described by the ratio $N_{0} / N$, where $N_{0}$ denotes the number of neutral atoms within the core, and $N$ is the total number of atoms and ions within the core. For comparison, unpublished predictions from our previous analysis [26] showed that, within untampered homogeneous clusters irradiated with photons of $32 \mathrm{~nm}$ wavelength, the distribution of ions of different charges peaks at the edge of the cluster. A similar observation has also been made in [27]. Therefore, the signal from an untampered cluster would be affected both by low scattering factors at the edge of the cluster and, for longer pulses, also by the fast expansion of the ions from the edge of the cluster. That is the reason why the idea of tampering is so promising, because covering the imaged object by a layer of another material could reduce these two damage factors. On the other side, the presence of tampering material leads to a partial loss of structural and orientational information on the imaged object. A dedicated analysis of this effect was performed in [28,29] and showed that a sufficiently thin tampering layer still allows the retrieval of sufficient structural information from the imaged object.

In this study we consider heterogeneous clusters composed of the noble gas atoms $\mathrm{Xe}$ and $\mathrm{Ar}$, which have been extensively studied experimentally [1-4,6-10]. Using a fully nonequilibrium kinetic equation code (i.e., the Boltzmann solver described in [26,30,31]), we will demonstrate the significant effect of cluster composition on the ionization dynamics. Predictions will be obtained for the wavelength of $32 \mathrm{~nm}$, which is available at the free-electron laser in Hamburg (FLASH) facility, but our conclusions can be qualitatively applied to samples of more complex structure and irradiated at shorter wavelength.

\section{MECHANISMS OF ELECTRONIC DAMAGE}

We have investigated ionization dynamics within heterogeneous spherically symmetric atomic clusters composed of xenon and argon atoms. For the simulations we have considered two cases: (i) a core consisting of $80 \mathrm{Xe}$ atoms that is covered with a layer composed of 70 to $1000 \mathrm{Ar}$ atoms, and (ii) a core consisting of $80 \mathrm{Ar}$ atoms that is covered with a layer composed of 70 to $1000 \mathrm{Xe}$ atoms. We mention here that the composition (i) was investigated experimentally at a different radiation wavelength $(13.7 \mathrm{~nm})$ at FLASH [6].

Our simulation tool is a Boltzmann solver [30] based on nonequilibrium kinetic equations. It includes contributions from photoionization, electron-impact ionization, electron elastic scattering, three-body recombination, and short-range electron-electron interactions. Ions and electrons could move during the simulations (simulation times were between 25 and $50 \mathrm{fs}$ ), although the displacement of ions at the considered pulse fluences was negligible $(<1 \AA)$.

The results of our simulations at a wavelength of $32 \mathrm{~nm}$ and at pulse fluences in the range from 1.25 to $2.50 \mathrm{~J} / \mathrm{cm}^{2}$ are shown in Fig. 1. For the simulations we were using rectangularly shaped pulses. We have checked that the FELinduced dynamics at this wavelength depends only on the pulse fluence and not on the temporal pulse shape. Dedicated simulations showed that the maximum difference in the obtained ion yield within the core was less than $7 \%$ for pulses of the same fluence but of different duration.

Figure 1(a) shows that a thicker Ar layer leads to an increase of the content of neutral $\mathrm{Xe}$ atoms within the Xe core after the FEL pulse has passed. In this case, tampering reduces the electronic damage within the tampered object. The damage reduction is weaker for irradiation with pulses of larger fluence. The impact of pulse fluence is more pronounced for thin tampering shells (with the number of atoms $<500$ ). For thick tampering shells it weakly changes with the pulse fluence.

In contrast, Fig. 1(b) shows that the Xe tamper leads to a decreased number of neutral atoms within the Ar core (stronger electronic damage) compared with the case of bare $\mathrm{Ar}_{80}$. There appears to be an almost constant vertical offset for the lowerfluence data relative to the highest-fluence data.

The striking differences between compositions (i) and (ii) can be explained as follows: During the irradiation of a sample, primary (photo-) and secondary (electronic) ionization processes occur and release electrons from atoms. Electrons are attracted by positively charged ions, and only the energetic electrons can leave the sample. With the increasing number of electrons emitted, the attractive Coulomb field inside the sample grows. It reduces the kinetic energy of subsequently 

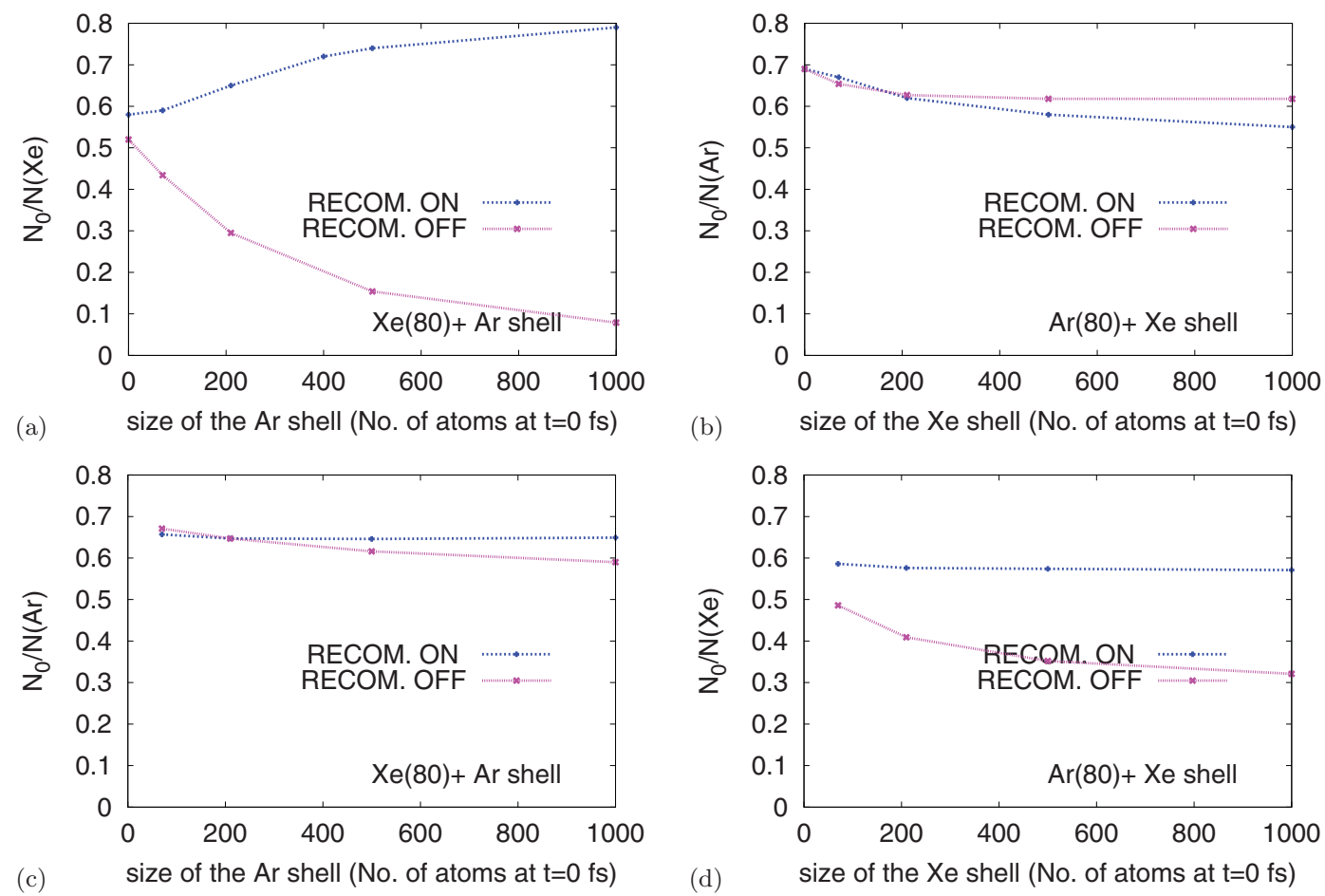

FIG. 2. (Color online) Content of neutral atoms within the Xe/Ar cores [(a), (b)] and shells [(c), (d)] as a function of the thickness of the tampering layer for [(a), (c)] $\mathrm{Xe}_{80}$ covered with an Ar layer, and [(b), (d)] $\mathrm{Ar}_{80}$ covered with an Xe layer. The pulses were temporally rectangular, with the pulse fluence of $F=2 \mathrm{~J} / \mathrm{cm}^{2}$. The results were obtained with Boltzmann solver simulations. During the simulation with recombination "switched on," electrons within the cluster could recombine through three-body recombination process. During the simulation with recombination "switched off" the electrons were (artificially) not allowed to recombine.

emitted electrons and eventually prevents further electron emission. Electrons become attracted toward the center of the system, leading to the generation of a thermally equilibrated nanoplasma at the core of the system. Stripped ions form a positively charged outer shell around the core. In the case of an untampered sample, the "net neutral core positively charged outer shell" structure is formed within the sample. In case of a tampered sample, the shell of positively charged ions is ideally located within the layer of the tampering material. Compared to the case of an untampered sample, the tampering shell produces additional electrons via photo- and secondary ionizations. The most energetic electrons escape from the sample but many electrons remain inside and are attracted toward the center of the system. This leads to further ionizations within the core. At the same time a competing inverse process occurs; namely, electronic three-body recombination. The rate for three-body recombination in Xe is about three times higher than in Ar. In the Ar core system the electrons from the Xe shell arriving at the Ar core further ionize the core. Therefore, there is an almost linear decrease of the neutral atom content within the Ar core with pulse fluence [Fig. 1(b)]. Neutral atom content within the Ar core decreases also with the thickness of the tampering shell, as a thicker shell produces more electrons that can enter the core and ionize it.

For the case of the $\mathrm{Xe}$ core, the picture is different. Recombination within the Xe core is efficient. Therefore, many electrons arriving from the tampering Ar shell at the Xe core can recombine there. However, this process equilibrates at a certain number of quasifree electrons within the Xe core. Therefore, the increase of the pulse fluence irradiating the Xe core system will not have as much influence on the neutral atom content within the Xe core above a certain thickness of the Ar shell [Fig. 1(a)].

In order to confirm the dominant role of three-bodyrecombination in differentiating the dynamics of heterogeneous systems, we have performed dedicated simulations at a pulse fluence of $2 \mathrm{~J} / \mathrm{cm}^{2}$, where we have artificially "switched off" three-body recombination within the core and the shell during the simulation (Fig. 2). We have observed that, for the Xe core system, this leads to efficient ionization of the core compared with the untampered sample, and the tampering effect of the outer shell completely disappears [Fig. 2(a)]. At the same time, "switching off" the recombination does not much affect the argon shell, and this almost independently of its size [Fig. 2(c)].

After switching off the recombination within the Ar core system, tampering with an Xe shell still leads to slightly higher damage within the Ar core compared with the case of an untampered sample [Fig. 2(b)]. The qualitative tendency from the case when recombination was "switched on" (thicker shell, higher damage) is maintained. However, this effect is now weaker than for the case with recombination switched on. In contrast, the xenon shell is more strongly affected: for the case of recombination "switched off," the ionization of the $\mathrm{Xe}$ shell is much higher than for the case with recombination "on" [Fig. 2(d)]. However, it depends only weakly on the shell thickness, compared with the case from Fig. 2(a).

These effects can be explained by studying the time evolution of electron densities within the Xe core and Ar core systems. The results reflect the fact that electronic processes in 

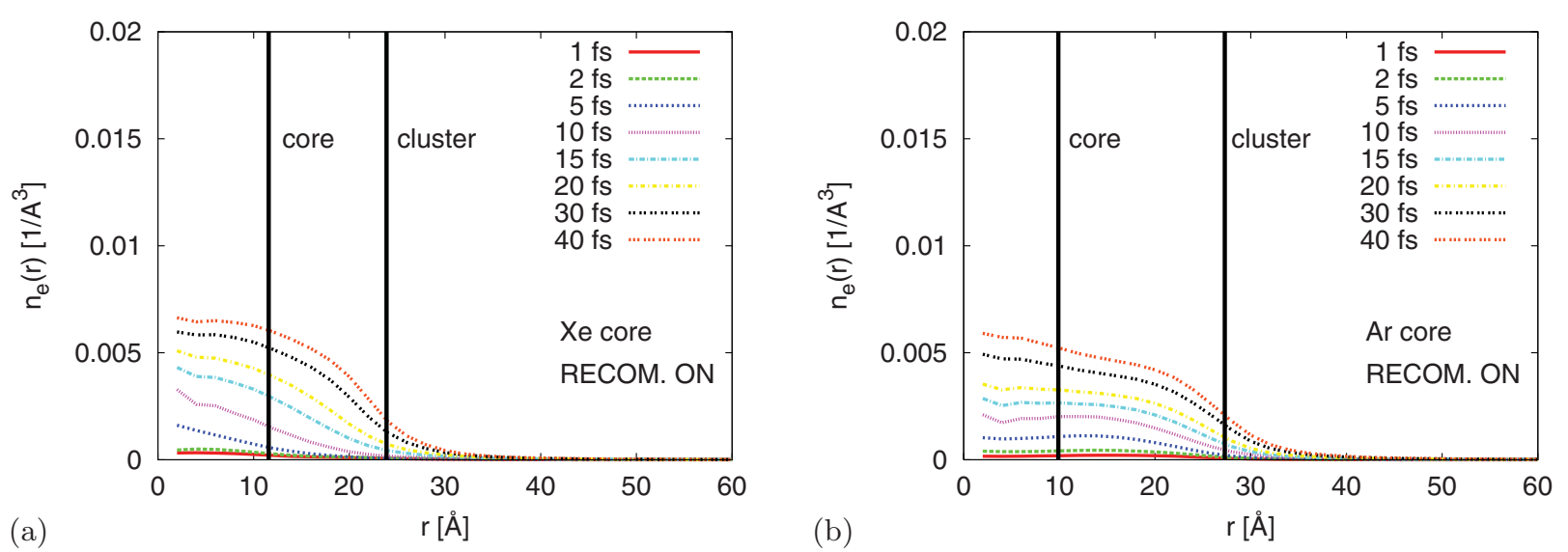

FIG. 3. (Color online) Time evolution of electron density within heterogeneous samples with (a) an $\mathrm{Xe}_{80}$ core covered with an Ar layer of $N=1000$ atoms and (b) an $\operatorname{Ar}_{80}$ core covered with an Xe layer of $N=1000$ atoms. The pulses were temporally rectangular, with a duration of $40 \mathrm{fs}$ and an intensity of $5 \times 10^{13} \mathrm{~W} / \mathrm{cm}^{2}$ (corresponding to a fluence of $F=2 \mathrm{~J} / \mathrm{cm}^{2}$ ). Vertical lines indicate core and total cluster radii. During this simulation (with recombination "switched on"), electrons within the cluster could recombine through three-body recombination.

Xe have significantly higher rates than in Ar. In Figs. 3 and 4, respectively, we compare the cases when electrons could and could not recombine during the simulation. "Switching off" the recombination for the case of the Xe core system leads to a significant increase of electron density within the Xe core [Fig. 4(a)] and, consequently, to an increase in the number of collisionally created ions within this core [Fig. 2(a)]. At the same time, the density of electrons in the Ar shell also increases. However, due to much lower recombination rates, this increase has almost no effect on the ionization state of the Ar shell [Fig. 2(c)]. For the case of the Ar core system, "switching off" the recombination also leads to an increase of the electron density within the Xe layer [Fig. 4(b)], resulting in more collisional ionizations within this shell [Fig. 2(d)]. The electron density within the Ar core remains comparable to that obtained with recombination "switched on." The explanation for this effect is that the most energetic electrons ionize collisionally within the tampering $\mathrm{Xe}$ shell, and the core becomes populated by electrons of lower energies that cannot further ionize. Therefore, the neutral atom content within the Ar core for the case of "recombination off" is slightly higher than for case of "recombination on" [Fig. 2(b)].

We have also performed an additional test, running the simulations with the recombination levels $n$, where $n$ denotes the principal quantum number, artificially set to be the same for $\mathrm{Xe}$ and Ar. The obtained results for the neutral atom content for $\mathrm{Xe}$ and Ar core systems are then very close to each other (not shown). This shows that the recombination levels differentiate the recombination dynamics with rates proportional to $n^{2}$.

The final conclusion is that the three-body recombination is predominantly responsible for the electronic tampering process at the considered radiation wavelength of $32 \mathrm{~nm}$. In order to explore the limits of the tampering method, we also present the results obtained for an $\mathrm{Xe}_{80}$ cluster covered with a shell consisting of $10^{6} \mathrm{Ar}$ atoms irradiated by an $F=1.25 \mathrm{~J} / \mathrm{cm}^{2}$ pulse. There is not much change in the tampering efficiency compared to the case with the shell consisting of $10^{3}$ Ar atoms $\left(N_{0} / N=0.80\right.$ for a $10^{3}$-atom $\mathrm{Ar}$
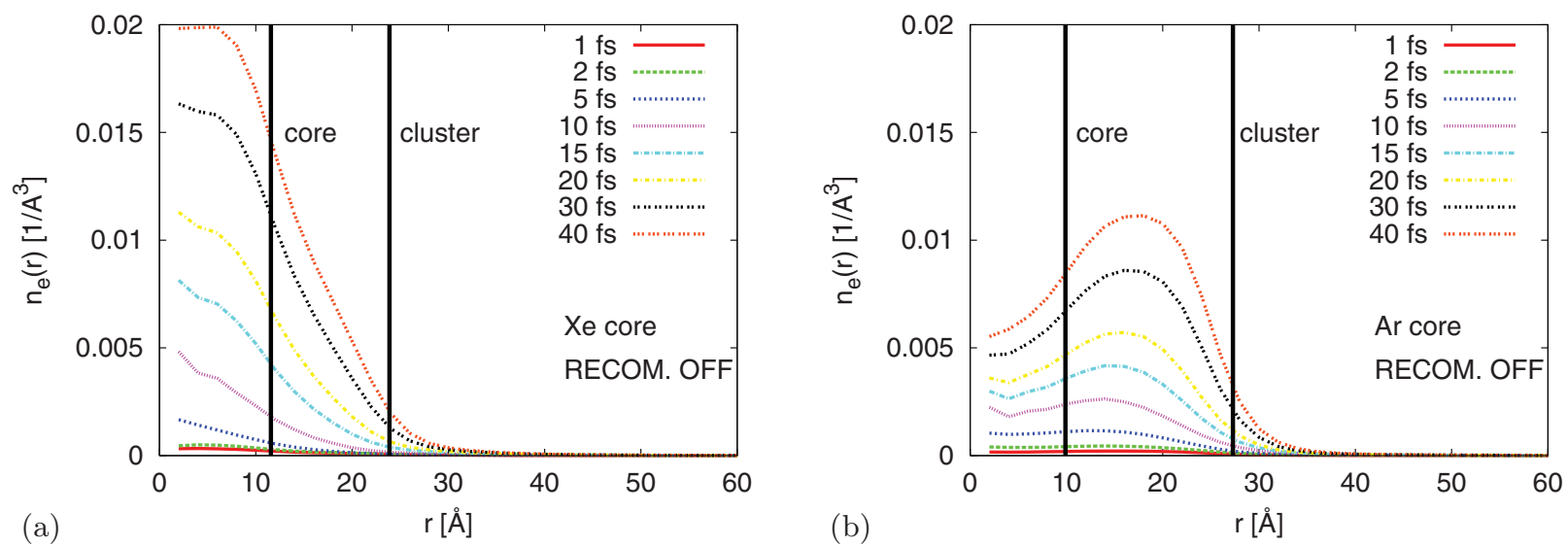

FIG. 4. (Color online) Time evolution of electron density within heterogeneous samples with (a) an $\mathrm{Xe}_{80}$ core covered with an Ar layer of $N=1000$ atoms, and (b) an $\mathrm{Ar}_{80}$ core covered with an Xe layer of $N=1000$ atoms. The pulses were temporally rectangular, with a duration of $40 \mathrm{fs}$ and an intensity of $5 \times 10^{13} \mathrm{~W} / \mathrm{cm}^{2}$ (corresponding to a fluence of $F=2 \mathrm{~J} / \mathrm{cm}^{2}$ ). Vertical lines indicate core and total cluster radii. During this simulation (with recombination "switched off") the electrons were (artificially) not allowed to recombine. 
shell and $N_{0} / N=0.82$ for a $10^{6}$-atom Ar shell). We would like to emphasize that the latter simulation was performed with the Boltzmann solver on a single computing core within two weeks. This demonstrates the high computational efficiency of our code, which can follow the dynamics within very large samples, which is hardly accessible with standard molecular dynamics.

In contrast to the hydrodynamic simulations performed so far $[19,22]$, which assumed that the electrons in the system reach instantaneously local thermodynamic equilibrium, our simulations are fully nonequilibrium: they follow the evolution of the electronic system during the pulse toward its equilibrium state.

Because the electronic processes determine the electronic tampering behavior of the outer layer, we expect that our results can be also qualitatively applied for complex samples irradiated with pulses of shorter wavelength and of a high fluence, at which the ionization progresses fast and destroys most of the chemical bonds early in the exposure. At shorter wavelength, the photoionization processes initiating the ionization dynamics will change, as core ionizations and subsequent Auger decays become accessible. Energetic electrons will be released; many of them will be able to leave the sample. If many electrons can leave, the density of electrons confined within the sample will be low. This will suppress three-body recombination. In such a case, then, as far as electronic tampering is concerned, the "Ar core scenario" might be realized, and tampering might in fact lead to an increase of electronic damage within the tampered sample.
This would require applying special techniques to retrieve the "undamaged" structural information from the recorded scattering pattern of the damaged object $[19,32]$.

\section{CONCLUSION}

In summary, our simulations, performed within the framework of a Boltzmann model that includes all predominant interactions and follows the nonequilibrium and equilibrium dynamics of irradiated samples, reveal the differing ionization scenarios for irradiation of heterogeneous clusters of different atomic composition. The physical mechanisms contributing to electronic damage are now understood and explained quantitatively. They can be qualitatively generalized to the case of clusters composed of different atomic species and irradiated with intense $\mathrm{X}$-ray pulses.

\section{ACKNOWLEDGMENTS}

Beata Ziaja is grateful to Thomas Fennel, Adrian Mancuso, and Karl-Heinz Meiwes-Broer for discussions and illuminating comments. Authors thank the colleagues from Dresden, especially Jan Michael Rost and Ulf Saalmann for fruitful discussions. This research was supported by the German Bundesministerium für Bildung und Forschung with Grants No. $05 \mathrm{KS} 4 \mathrm{KTC} / 1$ and No. $05 \mathrm{KS} 7 \mathrm{KT} 1$, by the DFG Project BO 3169/1-1, and by the Helmholtz Gemeinschaft, Impulsfond VH-VI-302. T.L. acknowledges DFG support under Grant No. LA 1431/2-3.
[1] T. Laarmann, A. R. B. deCastro, P. Gurtler, W. Laasch, J. Schulz, H. Wabnitz, and T. Moller, Nature (London) 420, 482 (2002).

[2] H. Wabnitz, Ph.D. thesis (unpublished), DESY-THESIS-2003026, 2003.

[3] T. Laarmann, M. Rusek, H. Wabnitz, J. Schulz, A. R. B. deCastro, P. Gurtler, W. Laasch, and T. Moller, Phys. Rev. Lett. 95, 063402 (2005).

[4] C. Bostedt et al., Phys. Rev. Lett. 100, 133401 (2008).

[5] T. Laarmann, A. R. B. deCastro, P. Gurtler, W. Laasch, J. Schulz, H. Wabnitz, and T. Moller, Phys. Rev. Lett. 92, 143401 (2004).

[6] M. Hoener et al., J. Phys. B 41, 181001 (2008).

[7] H. Thomas et al., J. Phys. B 42, 134018 (2009).

[8] C. Bostedt et al., J. Phys. B 43, 194011 (2010).

[9] C. Bostedt et al., New J. Phys. 12, 083004 (2010).

[10] H. Iwayama et al., J. Phys. B 42, 134019 (2009).

[11] B. F. Murphy, K. Hoffmann, A. Belolipetski, J. Keto, and T. Ditmire, Phys. Rev. Lett. 101, 203401 (2008).

[12] DESY. XFEL-info. DESY [http://xfelinfo.desy.de], 5:18-25 (2007).

[13] C. Bostedt et al., Nucl. Instr. Meth. Phys. Res. A 601, 108 (2009).

[14] T. Fennel et al., Rev. Mod. Phys. 82, 1793 (2010).

[15] R. Neutze et al., Nature (London) 406, 752 (2000).

[16] J. Miao, K. Hodgson, and D. Sayre, Proc. Natl. Acad. Sci. 98, 6641 (2001).

[17] Z. Jurek, G. Oszlányi, and G. Faigel, Europhys. Lett. 65, 491 (2004).
[18] S. P. Hau-Riege, R. A. London, G. Huldt, and H. N. Chapman, Phys. Rev. E 71, 061919 (2005).

[19] S. P. Hau-Riege, R. A. London, H. N. Chapman, A. Szoke, and N. Timneanu, Phys. Rev. Lett. 98, 198302 (2007).

[20] H. Chapman et al., Nature Phys. 2, 839 (2006).

[21] A. Mikaberidze, U. Saalmann, and J.-M. Rost, Phys. Rev. A 77, 041201 (2008).

[22] S. P. Hau-Riege et al., Phys. Rev. Lett. 104, 064801 (2010).

[23] C. Gnodtke, U. Saalmann, and J.-M. Rost, Phys. Rev. A 79, 041201 (2009).

[24] S. P. Hau-Riege, R. A. London, G. Huldt, and H. N. Chapman, Phys. Rev. E 69, 051906 (2004).

[25] Z. Jurek, G. Faigel, and M. Tegze, Eur. Phys. J. D 29, 217 (2004).

[26] B. Ziaja, T. Laarmann, H. Wabnitz, F. Wang, E. Weckert, C. Bostedt, and T. Möller, New J. Phys. 11, 103012 (2009).

[27] C. Siedschlag and J. M. Rost, Phys. Rev. Lett. 93, 043402 (2004).

[28] B. Ziaja et al., Ultramicroscopy 111, 793 (2011).

[29] F. Wang, E. Weckert, B. Ziaja, D. S. D. Larsson, and D. vanderSpoel, Phys. Rev. E 83, 031907 (2011).

[30] B. Ziaja, A. R. B. de Castro, E. Weckert, and T. Möller, Eur. Phys. J. D 40, 465 (2006).

[31] B. Ziaja, H. Wabnitz, F. Wang, E. Weckert, and T. Möller, Phys. Rev. Lett. 102, 205002 (2009).

[32] H. Quiney and K. Nugent, Nature Phys. 7, 142 (2011). 